\title{
Cardiac Image Fusion from Stand-Alone SPECT and CT: Clinical Experience
}

Oliver Gaemperli ${ }^{1}$, Tiziano Schepis ${ }^{1}$, Ines Valenta ${ }^{1}$, Lars Husmann ${ }^{2}$, Hans Scheffel ${ }^{2}$, Victor Duerst ${ }^{1}$, Franz R. Eberli ${ }^{1}$, Thomas F. Luscher ${ }^{1}$, Hatem Alkadhi ${ }^{2}$, and Philipp A. Kaufmann ${ }^{1,3}$

${ }^{I}$ Cardiovascular Center, University Hospital Zurich, Zurich, Switzerland; ${ }^{2}$ Institute of Diagnostic Radiology, University Hospital Zurich, Zurich, Switzerland; and ${ }^{3}$ Zurich Center for Integrative Human Physiology, University of Zurich, Zurich, Switzerland

Myocardial perfusion imaging with SPECT (SPECT-MPI) and 64slice CT angiography (CTA) are both established techniques for the noninvasive evaluation of coronary artery disease (CAD). Three-dimensional (3D) SPECT/CT image fusion may offer an incremental diagnostic value by integrating both sets of information. We report our first clinical experiences with fused 3D SPECT/CT in CAD patients. Methods: Thirty-eight consecutive patients with at least 1 perfusion defect on SPECT-MPI (1-d adenosine stress/rest SPECT with ${ }^{99 m}$ Tc-tetrofosmin) and 64slice CTA were included. 3D volume-rendered fused SPECT/ CT images were generated and compared with the findings from the side-by-side analysis with regard to coronary lesion interpretation by assigning the perfusion defects to their corresponding coronary lesion. Results: The fused SPECT/CT images added information on pathophysiologic lesion severity in 27 coronary stenoses $(22 \%)$ of 12 patients $(29 \%)(P<$ 0.001 ). Among 40 equivocal lesions on side-by-side analysis, the fused interpretation confirmed hemodynamic significance in 14 lesions and excluded functional relevance in 10 lesions. In 3 lesions, assignment of perfusion defect and coronary lesion appeared to be reliable on side-by-side analysis but proved to be inaccurate on fused interpretation. Added diagnostic information by SPECT/CT was more commonly found in patients with stenoses of small vessels $(P=0.004)$ and involvement of diagonal branches $(P=0.01)$. Conclusion: In addition to being intuitively convincing, 3D SPECT/CT fusion images in CAD may provide added diagnostic information on the functional relevance of coronary artery lesions.

Key Words: myocardial perfusion imaging; SPECT; coronary CT angiography; SPECT/CT fusion; coronary artery disease

J Nucl Med 2007; 48:696-703

DOI: 10.2967/jnumed.106.037606

\section{A}

precise, noninvasive technique for the diagnosis of coronary artery disease (CAD) should provide complementary information on coronary artery anatomy and pathophysiologic lesion severity (1-4). Although this can be

Received Oct. 25, 2006; revision accepted Feb. 12, 2007.

For correspondence or reprints contact: Philipp A. Kaufmann, MD, Nuclear Cardiology, Cardiovascular Center, University Hospital Zurich, NUK C 32, Raemistrasse 100, CH-8091 Zurich, Switzerland.

E-mail: pak@usz.ch

COPYRIGHT @ 2007 by the Society of Nuclear Medicine, Inc. achieved by mental integration of the information from coronary angiography (CA) and SPECT myocardial perfusion imaging (SPECT-MPI), standard myocardial distribution territories correspond in only 50\%-60\% with the real anatomic coronary tree (5). Several pioneering attempts of software-based image fusion from conventional CA and SPECT-MPI have been paving the way but were not implemented into clinical practice because its invasiveness precluded its use for noninvasive preinterventional decision making (5-8).

Recently, 64-slice CT angiography (CTA) has emerged as a truthful alternative to conventional $\mathrm{CA}$, with excellent diagnostic accuracy, in selected patients $(9,10)$. Combined with the advancements in fast-processing software for 3-dimensional (3D) reconstructions (11), this has allowed initial promising attempts of purely noninvasive SPECT/ $\mathrm{CT}$, directly relating individual myocardial wall territories to the subtending coronary artery (12-14).

The increasing interest in cardiac fusion imaging is currently raising the question of its clinical feasibility and usefulness. An evaluation seems all the more pertinent, as the integration of SPECT or PET scanners and high-end CT devices into hybrid scanners will promote the combined use of both techniques in the same patient. Alternatively, software solutions for fusion of SPECT or PET information with CTA may allow the combination of image sets obtained on 2 separate stand-alone scanners (14). The aim of the present study was to evaluate the added clinical value of cardiac 3D fusion imaging over the separate analysis of SPECT-MPI and 64-slice CTA on stand-alone scanners.

\section{MATERIALS AND METHODS}

\section{Study Population}

CTA and SPECT-MPI were performed on 180 consecutive patients $2 \pm 5 \mathrm{~d}$ apart. The reasons for each patient's referral were typical or atypical chest pain, pathologic treadmill test, preoperative risk stratification, or dyspnea. Of the 180 patients, only 38 with at least 1 fixed or reversible perfusion defect on SPECT-MPI were included, as cardiac fusion imaging is unlikely to provide any additional information in patients with normal SPECT-MPI. The study protocol was approved by the local institutional review board, and all patients gave written informed consent before enrollment. 


\section{SPECT-MPI}

All patients underwent a 1-d electrocardiographically (ECG)gated stress/rest protocol. A dose of $250-350 \mathrm{MBq}{ }^{99 \mathrm{~m}} \mathrm{Tc}-$ tetrofosmin was injected $3 \mathrm{~min}$ into the adenosine stress according to clinical practice (15). When contraindications for adenosine were present, dobutamine stress was used (16). This was followed by an injection of 3 times the stress dose at rest as recommended (17). Gated SPECT studies were acquired with a dual-head detector camera (Millenium VG and Hawkeye; GE Healthcare) using standard acquisition parameters as previously reported (1820 ) and x-ray-based attenuation correction $(21,22)$.

Image analysis was performed using a commercially available software package (Cedars QGS/QPS; Cedars-Sinai Medical Center, Los Angeles, CA) (23), and interpretation with regard to reversible (ischemia) and fixed (scar) defects was performed as previously reported (24). A fixed perfusion defect with normal regional wall motion on gated SPECT was considered an artifact rather than a scar (25). Anterior and septal wall perfusion defects were allocated to the left anterior descending coronary artery (LAD), lateral defects to the left circumflex coronary artery (LCX), and inferior defects to the right coronary artery (RCA) according to current clinical recommendations (26).

\section{4-Slice CTA}

CT was performed on a 64-detector CT scanner (LightSpeed VCT; GE Healthcare). First, a low-dose, prospectively ECG-gated native scan of the heart was performed for coronary calcium scoring. Scan parameters for CTA were detector collimation, $64 \times$ $0.625 \mathrm{~mm}$; total $z$-axis detector coverage, $40 \mathrm{~mm}$; gantry rotation time, $0.35 \mathrm{~s}$; tube voltage, $120 \mathrm{kV}$; ECG-modulated tube current, 280-750 mA; and pitch, 0.18-0.26, depending on the patient's heart rate. A bolus of $70-100 \mathrm{~mL}$ of contrast agent (Ultravist [Iopromide], $370 \mathrm{mg} / \mathrm{mL}$; Schering AG) was continuously injected into an antecubital vein $(50-80 \mathrm{~mL}$ at $5.0 \mathrm{~mL} / \mathrm{s}$, then $20 \mathrm{~mL}$ at $3.5 \mathrm{~mL} / \mathrm{s}$ ), and CT data acquisition was initiated as previously reported $(18,19)$. All patients with heart rates of $>65$ beats per minute (bpm) were pretreated intravenously with $5-15 \mathrm{mg}$ of metoprolol tartrate (Lopresor; Sankyo Pharma AG).

The CTA datasets were analyzed using axial source images, multiplanar reformations, and thin-slab maximum-intensity projections on a remote workstation (Advantage Workstation 4.3; GE Healthcare). The coronary tree was evaluated according to a 15segment model proposed by the American Heart Association (AHA) (27). The intermediary artery was designated as segment 16, if present, and considered to belong to the LAD. Every bypass graft was counted as an additional segment. Image quality was graded as excellent (no motion artifacts present), good (minor motion artifacts present), moderate (substantial motion artifacts present, but luminal assessment of significant stenoses still possible), heavily calcified (vessel lumen obscured by calcifications), and blurred (only contrast visualization inside the vessel possible) for the whole patient's dataset and for every coronary segment. Coronary lesions were visually assessed, and a diameter stenosis $\geq 50 \%$ was considered significant.

\section{Image Fusion}

Fusion of stress and rest SPECT-MPI with CTA images was performed on a designated workstation (Advantage workstation 4.3; GE Healthcare) using the CardIQ Fusion software package (GE Healthcare). All details of the fusion process have been extensively described, validated, and reported elsewhere (11). In brief, this software provides tools for optimal alignment of axial source SPECT and CT images using the myocardial outlines of SPECT and CT as landmarks and for SPECT image projection on the left ventricular (LV) epicardial surface. The window presets for the color scale projected on the LV epicardium are adopted from the corresponding separate SPECT images and remain unchanged during the fusion process. Additionally, the fusion software facilitates extraction of the aorta and the coronary vessel tree structure from the surrounding tissue. For better visualization of the septal wall, the right ventricle could be faded away by a cardiac transparency tool.

\section{Conventional CA}

Conventional CA was performed according to standard techniques, and the angiograms were evaluated in consensus by 2 experienced interventional cardiologists who were both unaware of the clinical history and the results from SPECT-MPI and CTA. The coronary artery tree was similarly subdivided according to the AHA guidelines (27), and each vessel segment was visually evaluated with regard to vessel border delineation on at least 2 different projections. A diameter stenosis $\geq 50 \%$ was considered significant. Coronary artery analysis was performed in all vessels with a diameter down to $1.5 \mathrm{~mm}$, including those vessels distal to complete occlusions.

\section{Side-by-Side Interpretation: SPECT Plus CT}

Two experienced nuclear cardiologists analyzed the nonfused SPECT-MPI and CTA images side-by-side with regard to functionally relevant coronary stenoses, and consensus was found in every patient. A lesion in any native or bypass graft vessel associated with a matching perfusion defect in its corresponding myocardial territory was considered functionally relevant. Furthermore, the CTA findings were compared with the results from invasive CA to ascertain CTA diagnostic accuracy. All nonevaluable segments were censored as positive, according to an intention-to-diagnose approach, and were included in the analysis.

\section{Fused Interpretation: SPECT/CT}

The fused 3D SPECT/CT image analysis was compared with the side-by-side analysis with regard to coronary lesion interpretation. First, the readers evaluated whether the fused SPECT/CT analysis provided any additional diagnostic information about the anatomic relationship between perfusion defects and coronary vessels. Additional diagnostic information was considered to be present if the fused images (a) modified the initial interpretation with regard to the relationship between the "culprit lesion" and the corresponding defect or (b) increased the diagnostic confidence for intermediate lesions or equivocal defects. Furthermore, a potential clinical utility was evaluated by retrospectively assessing whether the information increment provided by the fused analysis would have changed the patient's management (influence on revascularization strategy and target vessel selection).

\section{Statistical Analysis}

Statistical analysis was performed using SPSS Software version 12.0.1 for Windows (SPSS Inc.). All numeric variables are given as mean $\pm \mathrm{SD}$. Categoric data are given in percentages. A 2-tailed Student $t$ test was used for comparison of means. The findings from coronary lesion interpretation on side-by-side or fused interpretation were compared by applying the $\chi^{2}$ test. The Fisher exact test was used for comparison of the combined noninvasive findings with the target lesion on percutaneous coronary intervention. The results of CTA and conventional CA were compared 
by calculating the sensitivity, specificity, negative predictive value (NPV), and positive predictive value (PPV) of CTA for the detection of coronary stenoses on CA. Potential predictors for diagnostic increments on lesion interpretation by the fused analysis were determined using univariate and multivariate logistic regression analysis. The regression results are presented as odds ratios (OR) and their respective $95 \%$ confidence interval (CI). A 2 -sided $P$ value $<0.05$ was considered statistically significant for all tests.

\section{RESULTS}

Thirty-eight consecutive patients with at least 1 perfusion defect on SPECT-MPI were included in the study. The mean age of the study population was $67 \pm 9 \mathrm{y}$, and 9 patients were female (24\%). Table 1 summarizes each patient's clinical characteristics.

\section{SPECT-MPI Findings}

SPECT-MPI revealed 17 fixed, 19 reversible, and 7 mixed perfusion defects. Five patients (13\%) had perfusion defects in $>1$ coronary artery territory. The exact location of perfusion defects is listed in Table 2. Twenty-two defects were located in the anterior and septal wall, 12 in the inferior wall, and 9 in the lateral wall.

\section{4-Slice CTA Findings}

All patients were in stable sinus rhythm. Mean heart rate during the scan was $61 \pm 7 \mathrm{bpm}$. Image quality was excellent in 10 patients (26\%), good in 15 patients (39\%), moderate in 9 patients (24\%), heavily calcified in 2 patients $(5 \%)$, and blurred in 2 patients ( $5 \%$ ). The mean calcium score was $1,029 \pm 1,399$ Agatston score units. In total, 525 coronary segments in 152 coronary arteries were analyzed. A stenosis $\geq 50 \%$ was found in 75 evaluable segments (14\%). Eighteen

TABLE 1

Patient Characteristics $(n=38)$

\begin{tabular}{lr}
\multicolumn{1}{c}{ Characteristic } & $n(\%)$ \\
\hline Age $(\mathrm{y})$ & $67 \pm 9$ \\
Females $(n)$ & $9(24)$ \\
Body mass index $\left(\mathrm{kg} \times \mathrm{m}^{-2}\right)$ & $27.6 \pm 4.7$ \\
Medical history $(n)$ & \\
1-Vessel coronary disease & $6(16)$ \\
2-Vessel coronary disease & $4(11)$ \\
3-Vessel coronary disease & $7(18)$ \\
Previous myocardial infarction & $9(24)$ \\
Previous PCl & $14(37)$ \\
Previous CABG & $4(11)$ \\
Typical/atypical chest pain & $21(55)$ \\
Dyspnea & $9(24)$ \\
Pathologic exercise test & $11(29)$ \\
Cardiovascular risk factors $(n)$ & $8(21)$ \\
Diabetes mellitus & $23(61)$ \\
Hypertension & $28(74)$ \\
Dyslipidemia & $19(50)$ \\
Current smokers &
\end{tabular}

$\mathrm{PCl}=$ percutaneous coronary intervention; $\mathrm{CABG}=$ coronary artery bypass grafting. coronary segments $(3 \%)$ were qualified as blurred, 17 segments (3\%) were heavily calcified, and 14 segments (3\%) had intracoronary stents that rendered a reliable luminal evaluation impossible. According to an intention-to-diagnose strategy, all nonevaluable segments were censored as positive, resulting in 124 stenosed coronary segments (Table 2). The remaining 401 coronary segments (76\%) were normal or showed only insignificant wall alterations.

\section{Comparison of CTA Versus Conventional CA}

In 25 of the 38 patients (66\%) a conventional CA was performed within $26 \pm 25 \mathrm{~d}$ of the CTA study. In these 25 patients, 339 coronary segments in 100 coronary arteries were analyzed on CTA. Forty-five segments (13\%) showed stenoses $\geq 50 \%$ on CTA. Thirty-eight segments $(11 \%)$ were not evaluable due to blurring $(n=16)$, heavy calcifications ( $n=$ $13)$, or intracoronary stents $(n=9)$. On a per-segment basis, sensitivity, specificity, NPV, and PPV of CTA for the detection of stenoses $\geq 50 \%$ on CA were $81 \%, 98 \%, 97 \%$, and $87 \%$, respectively, when all nonevaluable segments were excluded. For the intention-to-diagnose analysis, the latter were included, resulting in a sensitivity, specificity, NPV, and PPV of $85 \%, 88 \%, 97 \%$, and $60 \%$, respectively.

\section{Findings of Side-by-Side Analysis}

On side-by-side analysis of SPECT-MPI and CTA, 18 coronary stenoses $(15 \%)$ were assigned to their corresponding perfusion defect with a high level of confidence (Table 2) and, therefore, were considered functionally relevant (Fig. 1). Conversely, 66 coronary stenoses (53\%) were not associated with perfusion defects and, therefore, were classified as hemodynamically irrelevant. The remaining 40 segments with stenoses (32\%) (in 17 patients) were considered equivocal, as the side-by-side analysis did not allow exclusion of hemodynamic significance in these segments. In 4 patients no coronary stenoses were found on CTA and this was confirmed later by conventional CA; therefore, the SPECT results were considered false-positive because of either complete left bundle branch block $(n=1)$ or attenuation artifacts $(n=3)$.

\section{Findings of SPECT/CT Fusion}

Fusion of CTA and SPECT-MPI was successfully performed in all patients. Of the 40 equivocal coronary lesions on side-by-side analysis, 14 were associated with a perfusion defect in its corresponding coronary artery territory and, therefore, were considered hemodynamically relevant. In contrast, in 10 coronary lesions the fused analysis definitely allowed exclusion of hemodynamic significance. The remaining 16 lesions were 8 pairs of sequential neighboring lesions, for which a clear identification of the culprit lesion must remain inconclusive, although the vessel was reliably identified. In 3 lesions assignment of perfusion defect and coronary lesion appeared to be trustworthy on side-by-side analysis but turned out to be inaccurate on the fused analysis (hemodynamically relevant, $n=2$; irrelevant, $n=1$; patients 22 and 30). Thus, the fused SPECT/CT 
TABLE 2

Results of Nonfused and Fused SPECT/CT Analysis

\begin{tabular}{|c|c|c|c|c|c|c|c|}
\hline \multirow{2}{*}{$\begin{array}{l}\text { Patient } \\
\text { no. }\end{array}$} & \multirow[b]{2}{*}{ Sex } & \multirow{2}{*}{$\begin{array}{c}\text { Age } \\
(y)\end{array}$} & \multirow{2}{*}{$\begin{array}{c}\text { CTA results: } \\
\text { coronary segment } \\
\text { with stenosis }>50 \%\end{array}$} & \multicolumn{2}{|c|}{ MPI results: perfusion defect } & \multicolumn{2}{|c|}{ Culprit vessel: analysis } \\
\hline & & & & Reversible & Fixed perfusion & Separate nonfused & Fused \\
\hline 1 & M & 48 & $2,7,9,11$ & & Inferior & Mid RCA & Mid RCA \\
\hline 2 & M & 65 & - & & $\begin{array}{l}\text { Inferior, } \\
\text { anterior }\end{array}$ & None & None \\
\hline 3 & M & 67 & 11,13 & & Lateral & Prox LCX & Prox LCX \\
\hline 4 & $\mathrm{M}$ & 61 & $2,6,10,11,16$ & Anterior & & Prox LAD/DA2/IM* & DA2 \\
\hline 5 & M & 61 & 6,9 & & Anterior & Prox LAD/DA1* & DA1 \\
\hline 6 & M & 50 & 6,7 & Anteroapical & & Prox/mid LAD* & Prox/mid LAD* \\
\hline 7 & M & 62 & $2,3,4,7,9$ & Anterior & Posterior & Mid LAD, mid RCA & Mid LAD, mid RCA \\
\hline 8 & M & 76 & $1,6,8,9,10,11,13$ & & Inferior & Prox RCA & Prox RCA \\
\hline 9 & M & 74 & $1,2,3,4,7,8,9,14$ & Apical & & Mid/dist LAD/DA1* & Mid/dist LAD* \\
\hline 10 & M & 63 & 7,10 & Apical & & Mid LAD/DA2* & DA2 \\
\hline 11 & M & 79 & $1,2,7,9$ & Inferoapical & & Prox/mid RCA* & Prox/mid RCA* \\
\hline 12 & $\mathrm{~F}$ & 63 & $1,2,6$ & & Posterior & Prox/mid RCA* & Prox/mid RCA* \\
\hline 13 & $\mathrm{~F}$ & 67 & - & Anteroapical & & None & None \\
\hline 14 & $\mathrm{~F}$ & 76 & $7,11,13,14$ & Laterobasal & & Mid LCX & Mid LCX \\
\hline 15 & $\mathrm{~F}$ & 78 & 2,3 & Anterior & Anterior & None & None \\
\hline 16 & M & 60 & - & & Inferoapical & $\mathrm{PDA}^{\dagger}$ & PDA \\
\hline 17 & $\mathrm{~F}$ & 56 & $1,7,8,12$ & Apical & & Mid/dist LAD* & Mid/dist LAD* \\
\hline 18 & $\mathrm{~F}$ & 59 & 12 & Lateral & Lateral & $\mathrm{OM}$ & $\mathrm{OM}$ \\
\hline 19 & M & 66 & $\begin{array}{l}\text { 1, 2, 4, 6, 7, 11, 13, 15, } \\
\text { RIMA, VG1, VG2 }\end{array}$ & Inferior & Inferior & VG2 & VG2 \\
\hline 20 & M & 68 & $2,3,6,11,13$ & & Inferior & Prox RCA & Prox RCA \\
\hline 21 & $\mathrm{~F}$ & 86 & 7,10 & Apical & & Mid LAD/DA2* & DA2 \\
\hline 22 & M & 74 & $2,8,98,12,16$ & $\begin{array}{l}\text { Inferoapical, } \\
\text { anterolateral }\end{array}$ & & $\mathrm{DA} 1, \mathrm{OM}^{\star}$ & DA1, IM \\
\hline 23 & M & 75 & 7 & & Apical & qMid LAD & Mid LAD \\
\hline 24 & $\mathrm{~F}$ & 77 & 7,9 & & Anteroapical & Mid LAD/DA1* & Mid LAD \\
\hline 25 & M & 57 & 6,13 & Posterior & & Dist LCX & Dist LCX \\
\hline 26 & M & 69 & 9 & Anteroapical & & Mid LAD/DA1* & DA1 \\
\hline 27 & M & 50 & 6 & Anteroseptal & Anteroseptal & Prox LAD & Prox LAD \\
\hline 28 & M & 61 & $6,8,10,11,12,13$ & Anterior, lateral & Anterior & LAD/DA2, LCX/OM* & Dist LAD, DA2, OM \\
\hline 29 & M & 74 & $1,2,3,6,7$ & & Apical & Prox/mid LAD* & Prox/mid LAD* \\
\hline 30 & M & 77 & $1,2,5,6,7,11,13,16$ & & Inferolateral & Mid LCX & Mid LCX, IM \\
\hline 31 & M & 71 & 6 & Apical & & Prox LAD & Prox LAD \\
\hline 32 & $\mathrm{M}$ & 70 & $1,2,3,8,11,13, \mathrm{VG} 1$ & & Inferolateral & Prox RCA/prox LCX* & Prox RCA, prox LCX \\
\hline 33 & $\mathrm{~F}$ & 75 & $1,3,6,7$ & Apical & Inferior & LAD, prox/mid RCA* & LAD, prox/mid RCA* \\
\hline 34 & M & 66 & 7,9 & Apical & & Mid LAD/DA1* & DA1 \\
\hline 35 & M & 55 & 1 & Inferoseptal & Inferoseptal & Prox RCA & Prox RCA \\
\hline 36 & M & 69 & $1,4,9,13$ & Lateral & & Mid LCX & Mid LCX \\
\hline 37 & M & 56 & - & & Anteroseptal & None & None \\
\hline 38 & M & 78 & 2 & Inferior & Inferior & Mid RCA & Mid RCA \\
\hline
\end{tabular}

*Denotes insufficient diagnostic confidence for identification of hemodynamically relevant coronary lesion.

${ }^{\dagger}$ Status post percutaneous transluminal coronary angioglasty without stenting; no residual stenosis on CTA.

Mid = middle; prox = proximal; DA2 = second diagonal artery; IM = intermediary branch; DA1 = first diagonal artery; dist = distal; $\mathrm{PDA}=$ posterior descending artery; OM = obtuse marginal branch; RIMA = right internal mammary artery; VG1 = vein graft 1 ; VG2 = vein graft 2; PLA = posterolateral artery.

Numbers for coronary segments are coded according to a modified coronary segment model proposed by the AHA (27): $1=$ proximal RCA; $2=$ mid RCA; $3=$ distal RCA; $4=$ PDA; $5=$ LMA (left main coronary artery); $6=$ proximal LAD; $7=$ mid LAD; $8=$ distal LAD; $9=$ DA1; 10 = DA2; 11 = proximal LCX; $12=$ OM; 13 = mid LCX; $14=$ first posterolateral artery; $15=$ second posterolateral artery; $16=$ IM.

interpretation provided added information with regard to 27 lesions in 12 patients and allowed exclusion of a relevant lesion in significantly more segments than the side-by-side analysis $(P<0.001)$ (Fig. 1). Figures $2-4$ show examples of these patients, and an overview of all findings with the different modalities is given in Table 2.

\section{Comparison of Fused and Side-by-Side Analysis for Detection of Coronary Lesions Requiring Revascularization}

In $10(40 \%)$ of 25 patients undergoing conventional CA, a percutaneous coronary intervention (PCI) with stent implantation involving the following vessels was performed: LAD 


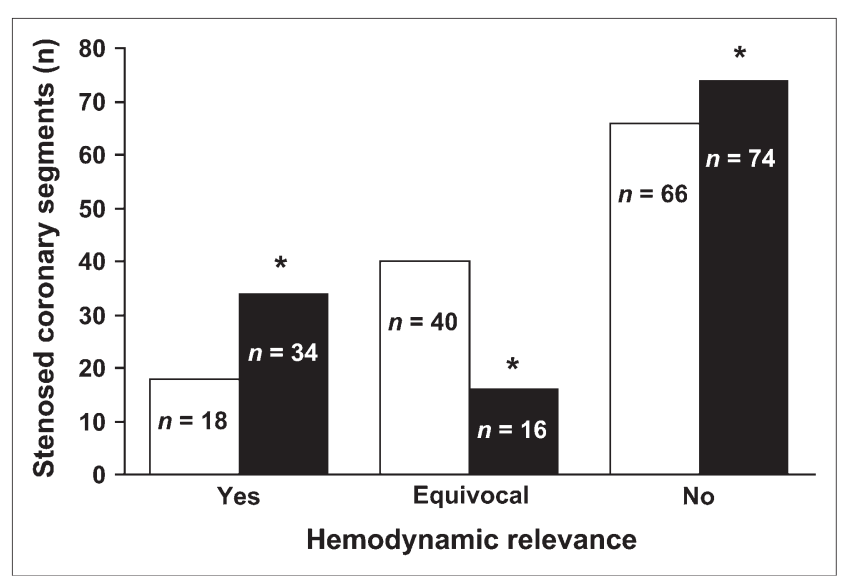

FIGURE 1. Interpretation of stenosed coronary segments with regard to hemodynamic significance on side-by-side (white columns) or fused (black columns) analysis. ${ }^{*} P<0.001$ for comparison of fused vs. side-by-side analysis ( $\chi^{2}$ test).

$(n=6)$, diagonal artery (DA) $(n=2), \operatorname{LCX}(n=2)$, obtuse marginal branch $(n=1)$, and RCA $(n=2)$. When patients were evaluated using only side-by-side analysis, a discrepancy was found between the coronary lesion treated as the culprit lesion with PCI and the functionally relevant lesion on initial noninvasive testing in 2 patients (patients 5 and 21). Fused SPECT/CT analysis revealed such a discrepancy in 2 additional patients (patients 4 and 34) with a stenosis of the LAD and DA (on CTA and CA) (Fig. 4) ( $P$ = not significant). Although the ischemic perfusion defect could be allocated to a DA using the SPECT/CT fusion, the LAD lesion was treated percutaneously on CA in both patients (Fig. 4).

\section{Predictors of Incremental Diagnostic Information of SPECT/CT Fusion}

Patients with incremental diagnostic information on SPECT/CT fusion $(n=12)$ had significant stenoses in small coronary arteries $(2.1 \pm 0.5$ vs. $3.3 \pm 0.7 \mathrm{~mm}, P<$ 0.001). Diagonal branches were more commonly involved ( $83 \%$ vs. $35 \%, P=0.01$ ), and calcium scores tended to be higher $(1,439 \pm 1,939$ vs. $840 \pm 1,060, P=0.23)$. Significant predictors (by univariate regression analysis) for incremental diagnostic information of SPECT/CT fusion were the diameter of the culprit vessel $(P=0.004)$ and the involvement of diagonal branches $(P=0.01)$ (Table 3$)$. When using multivariate logistic regression, only the former parameter reached the level of statistical significance (OR, 0.018 [95\% CI, 0.001-0.944], $P=0.047$ ).

\section{DISCUSSION}

Our results demonstrate that in almost one third of patients undergoing combined SPECT-MPI and CTA studies, the fused SPECT/CT analysis provided added diagnostic information on pathophysiologic lesion severity not obtained on side-by-side analysis. The incremental value was most pronounced for functionally relevant lesions in distal segments and diagonal branches and in vessels with extensive CAD or heavy calcification on CTA. The individual variance of coronary artery anatomy and the complex disease pattern in these patients allowed correct
FIGURE 2. (A) Stress and rest perfusion polar maps of SPECT-MPI study show mixed basal anterolateral defect and reversible inferoapical perfusion defect (arrowheads). (B and D) Fused SPECT/ $C T$ images reveal total occlusion of LAD and subtotal occlusion of first diagonal branch (DA1), which are confirmed by conventional CA (C). Anterolateral perfusion defect is caused by lesion of partially calcified small intermediary branch (IM); however, this vessel is not well visualized by $\mathrm{CA}$.

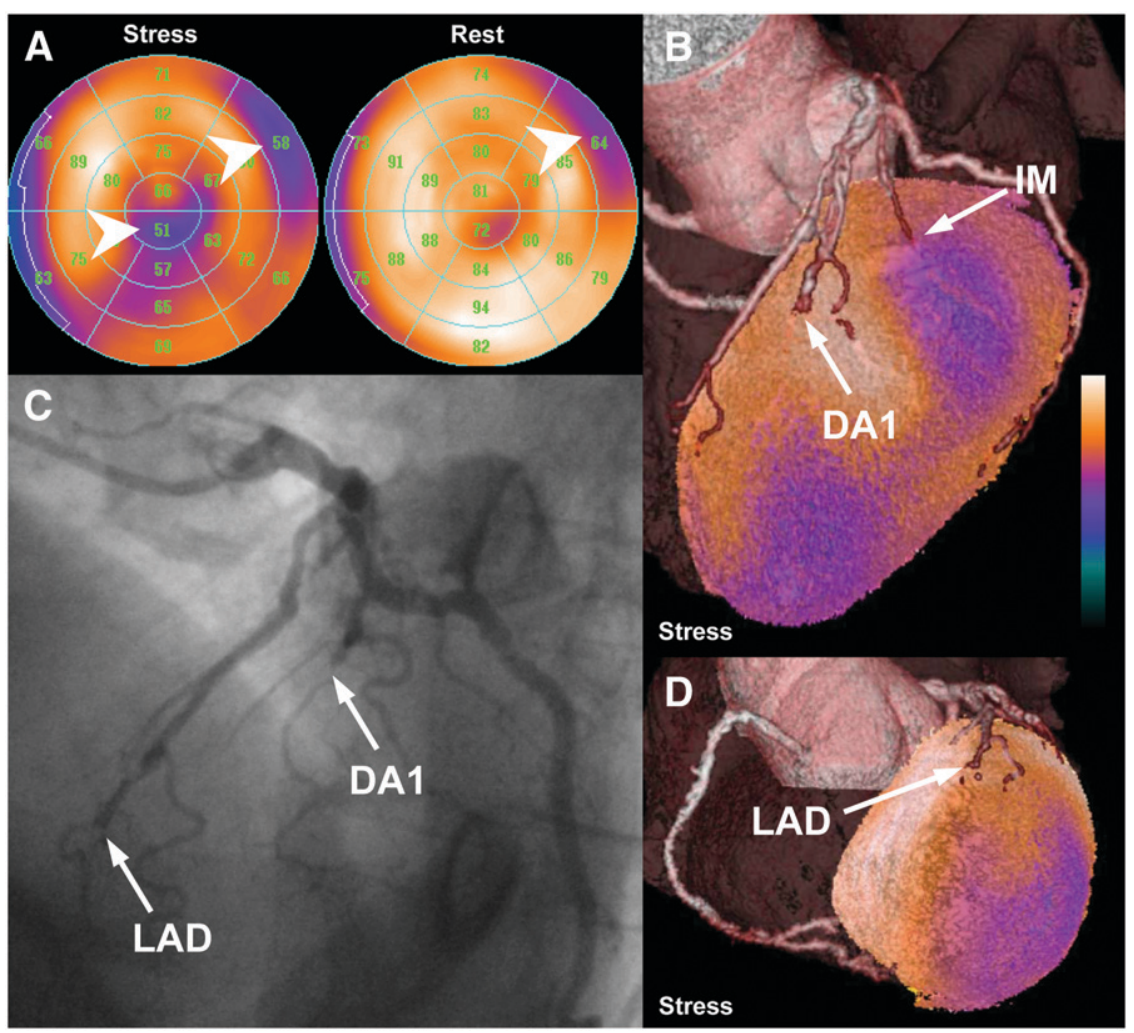




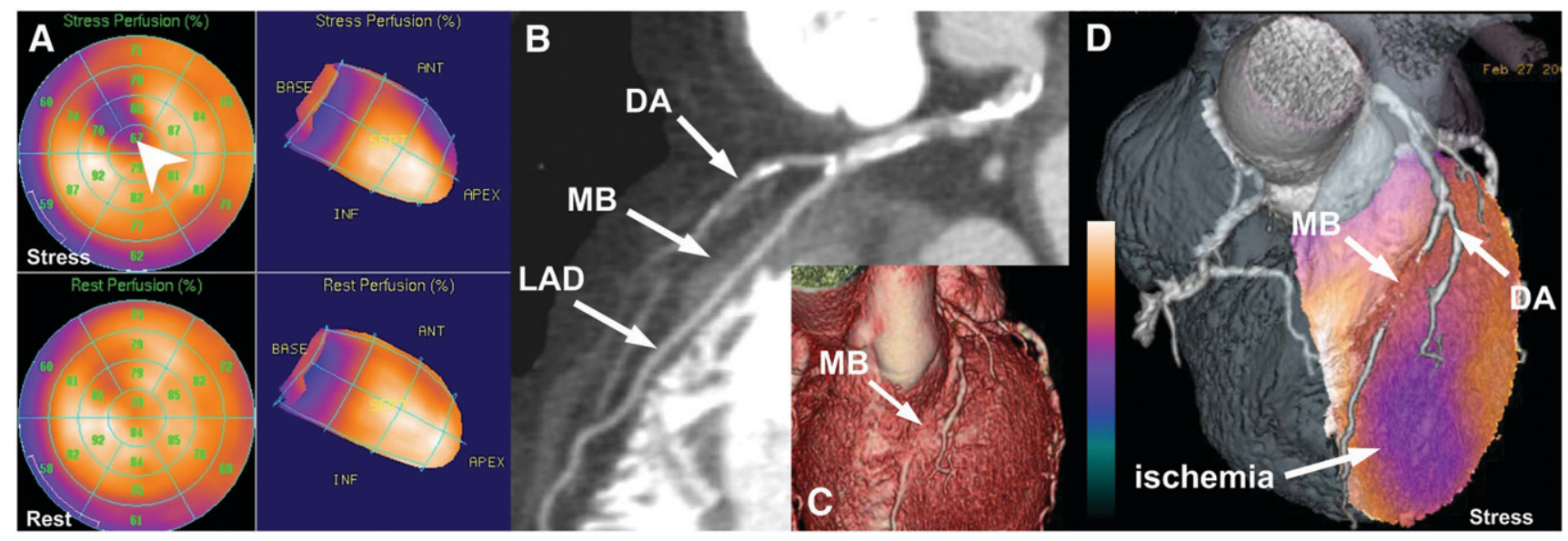

FIGURE 3. (A) Perfusion polar maps at stress (dobutamine stress) and rest show reversible anteroseptal perfusion defect. (B and C) 64-slice CTA revealed myocardial bridging (MB) of mid LAD of $>2-\mathrm{cm}$ length and calcified plaque at origin of first diagonal branch (DA). (D) Fused 3D SPECT/CT images could allocate reversible perfusion defect to DA, whereas MB seemed to be hemodynamically insignificant.

allocation of perfusion defect and subtending coronary artery only by fusion of SPECT-MPI and CTA.

It is well established that a comprehensive assessment of CAD requires not only morphologic information about coronary artery stenosis location and degree but also functional information on pathophysiologic lesion severity $(2,3)$. Eventually, many factors that cannot fully be assessed with coronary luminology determine whether a given stenosis really induces a myocardial perfusion defect. Therefore, an evaluation of the functional relevance of coronary stenosis is generally recommended to guide further decisions about revascularization strategies (3). Software-based 3D image fusion of SPECT-MPI and CT datasets offers superior diagnostic information with regard to identification of the culprit vessel and increases diagnostic confi- dence for categorizing intermediate lesions and equivocal perfusion defects. In fact, most of the lesions that were originally found to be equivocal with regard to pathophysiologic severity on side-by-side analysis - as they could not be firmly assigned to a perfusion defect-were classified with high confidence on the fused analysis. Thus, the greatest added value appears to be the firm exclusion of hemodynamic significance of coronary abnormalities seen on CTA, which might help to avoid unnecessary interventional procedures.

However, the clinical usefulness in terms of impact on patients' management of cardiac SPECT/CT fusion may not be as high as expected for several reasons. First, one third of the patients with incremental information by fused SPECT/CT had fixed perfusion defects, which generally would not justify revascularization without evidence of

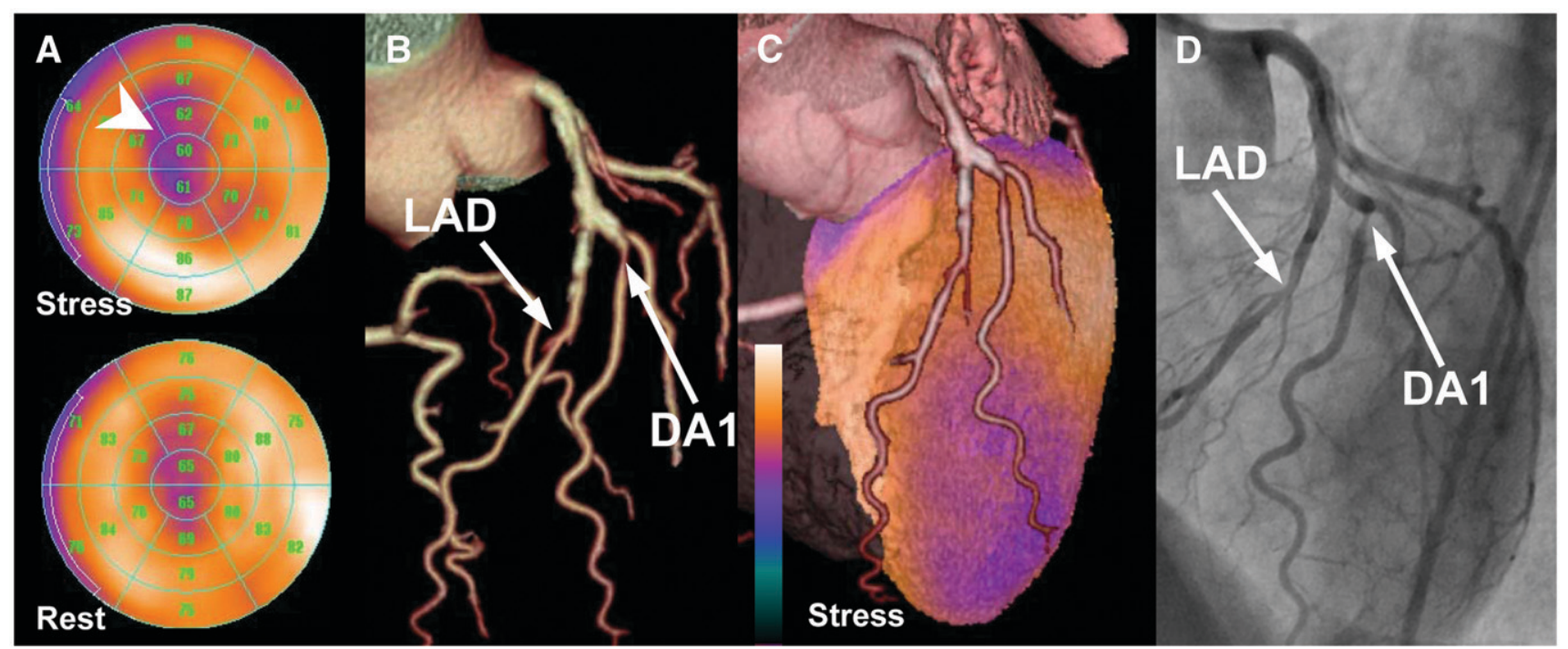

FIGURE 4. (A) Perfusion polar maps of SPECT-MPI at stress and rest show largely reversible anteroapical perfusion defect (arrowhead). (B) 3D volume-rendered CTA images show coronary vessel tree with stenosis of mid LAD and proximal stenosis of first diagonal branch (DA1). (C) Fused 3D SPECT/CT images are able to identify DA1 stenosis as functionally relevant lesions. (D) Findings were confirmed by invasive CA. 
TABLE 3

Predictors of Diagnostic Increment Using 3D SPECT/CT Fusion*

\begin{tabular}{llc}
\multicolumn{1}{c}{ Predictor } & OR $(95 \% \mathrm{Cl})$ & $P$ value \\
\hline Diameter of vessel with functionally relevant stenosis $(\mathrm{mm})$ & $0.05(0.01-0.39)$ & 0.004 \\
Involvement of diagonal branches & $9.44(1.69-52.73)$ & 0.01 \\
Calcium score (log, Agatston score) & $1.38(0.81-2.33)$ & $\mathrm{NS}$ \\
Number of vessels involved & $1.18(0.57-2.45)$ & $\mathrm{NS}$ \\
Image quality heavily calcified or blurred & $0.88(0.47-1.67)$ & $\mathrm{NS}$ \\
Mean heart rate at scan (bpm) & $0.99(0.90-1.10)$ & $\mathrm{NS}$ \\
Perfusion defect extent & $1.01(0.95-1.08)$ & $\mathrm{NS}$ \\
& & \\
\hline *By univariate logistic regression analysis. & & \\
$\mathrm{NS}=$ not significant. & & \\
\hline
\end{tabular}

viability, which was not assessed in the present study. Second, SPECT/CT fusion proved to be useful for lesions in distal vessels and side branches with limited prognostic impact, regardless of revascularization strategy (28). Similarly, for small perfusion defects, conservative management may be justified without the need for invasive procedures (29). However, for the treatment of refractory angina in patients with multiple intermediate lesions, image fusion may nevertheless facilitate identification and appropriate revascularization of the culprit lesion.

From a computational perspective, a hardware-based approach to image fusion using hybrid scanners seems superior to a software-based approach, as it permits the acquisition of coregistered anatomic and functional images (30). At a software level, this is achieved through the use of landmark-, surface-, or intensity-based coregistration techniques. Most likely it will depend on the individual setting of each institution to determine the type of approach-that is, software-based fusion or hybrid scanner-that is best tailored for its particular purpose.

Several limitations are noteworthy. As 3D SPECT/CT fusion was analyzed retrospectively, its impact on patient management could not be prospectively assessed. In addition, no true gold standard is available to validate the discrepancies observed between the findings on SPECT/CT and the decision by the interventional cardiologist to revascularize, although the accuracy of the fusion process has been recently documented (11).

Furthermore, conventional CA was only performed in two thirds of the patients. However, in these patients the findings from CTA and CA correlated well and the diagnostic accuracy of the CTA images was within the range of previously published data $(9,10)$. The rate of nonevaluable segments was slightly higher in the present study compared with the latter reports. This finding was due to the relatively high calcium score as a result of the inclusion criteria (perfusion defect), setting them at a high likelihood of CAD. New CT technology may enable scanning of patients with high coronary calcium burden or high heart rates without loss of image quality (31).

The combination of SPECT-MPI with a ${ }^{99 \mathrm{~m} T c-l a b e l e d}$ compound and CTA results in a radiation exposure of 15-
$25 \mathrm{mSv}$, comparable to the exposure from a thallium scan. As the fusion per se may increase the information obtained from each separate scan at no additional cost of radiation, its use may be welcomed as an adjunct to SPECT-MPI similar to the introduction of gating.

\section{CONCLUSION}

Software-based 3D cardiac SPECT/CT fusion imaging provides additional information about hemodynamic relevance and facilitates lesion interpretation by allowing exact allocation of perfusion defects to its subtending coronary artery.

\section{ACKNOWLEDGMENTS}

We are grateful to Ratko Milovanovic, our deputy chief radiographer, for his excellent technical support. This study was supported by a grant from the Swiss National Science Foundation (grant PP00A-68835) and by a grant from the National Center of Competence in Research, Computer Aided and Image Guided Medical Interventions of the Swiss National Science Foundation.

\section{REFERENCES}

1. Namdar M, Hany TF, Koepfli P, et al. Integrated PET/CT for the assessment of coronary artery disease: a feasibility study. J Nucl Med. 2005;46:930-935.

2. Klocke FJ, Baird MG, Lorell BH, et al. ACC/AHA/ASNC guidelines for the clinical use of cardiac radionuclide imaging: executive summary - a report of the American College of Cardiology/American Heart Association Task Force on Practice Guidelines (ACC/AHA/ASNC Committee to Revise the 1995 Guidelines for the Clinical Use of Cardiac Radionuclide Imaging). Circulation. 2003;108: 1404-1418.

3. Silber S, Albertsson P, Aviles FF, et al. Guidelines for percutaneous coronary interventions: the Task Force for Percutaneous Coronary Interventions of the European Society of Cardiology. Eur Heart J. 2005;26:804-847.

4. Smith SC Jr, Feldman TE, Hirshfeld JW Jr, et al. ACC/AHA/SCAI 2005 guideline update for percutaneous coronary intervention: a report of the American College of Cardiology/American Heart Association Task Force on Practice Guidelines (ACC/AHA/SCAI Writing Committee to Update 2001 Guidelines for Percutaneous Coronary Intervention). Circulation. 2006;113: $156-175$.

5. Schindler TH, Magosaki N, Jeserich M, et al. Fusion imaging: combined visualization of $3 \mathrm{D}$ reconstructed coronary artery tree and 3D myocardial scintigraphic image in coronary artery disease. Int J Card Imaging. 1999;15: 357-368; discussion, 369-370. 
6. Peifer JW, Ezquerra NF, Cooke CD, et al. Visualization of multimodality cardiac imagery. IEEE Trans Biomed Eng. 1990;37:744-756.

7. Nishimura Y, Fukuchi K, Katafuchi T, et al. Superimposed display of coronary artery on gated myocardial perfusion scintigraphy. J Nucl Med. 2004;45:1444-1449.

8. Faber TL, Santana CA, Garcia EV, et al. Three-dimensional fusion of coronary arteries with myocardial perfusion distributions: clinical validation. J Nucl Med. 2004;45:745-753.

9. Leschka S, Alkadhi H, Plass A, et al. Accuracy of MSCT coronary angiography with 64-slice technology: first experience. Eur Heart J. 2005;26:1482-1487.

10. Raff GL, Gallagher MJ, O'Neill WW, Goldstein JA. Diagnostic accuracy of noninvasive coronary angiography using 64-slice spiral computed tomography. J Am Coll Cardiol. 2005;46:552-557.

11. Gaemperli O, Schepis T, Kalff V, et al. Validation of a new cardiac image fusion software for three-dimensional integration of myocardial perfusion SPECT and stand-alone 64-slice CT angiography. Eur J Nucl Med Mol Imaging. January 24, 2007 [Epub ahead of print].

12. Nakaura T, Utsunomiya D, Shiraishi S, et al. Three-dimensional cardiac image fusion using new CT angiography and SPECT methods. AJR. 2005;185:1554-1557.

13. Nakaura T, Utsunomiya D, Shiraishi S, et al. Images in cardiovascular medicine: fusion imaging between myocardial perfusion single photon emission computed tomography and cardiac computed tomography. Circulation. 2005;112:e47-e48.

14. Gaemperli O, Schepis T, Kaufmann PA. SPECT-CT fusion imaging integrating anatomy and perfusion. Eur Heart J. 2007;28:145.

15. Cerqueira MD, Verani MS, Schwaiger M, Heo J, Iskandrian AS. Safety profile of adenosine stress perfusion imaging: results from the Adenoscan Multicenter Trial Registry. J Am Coll Cardiol. 1994;23:384-389.

16. Elhendy A, Bax JJ, Poldermans D. Dobutamine stress myocardial perfusion imaging in coronary artery disease. J Nucl Med. 2002;43:1634-1646.

17. Hesse B, Tagil K, Cuocolo A, et al. EANM/ESC procedural guidelines for myocardial perfusion imaging in nuclear cardiology. Eur J Nucl Med Mol Imaging. 2005;32:855-897.

18. Gaemperli O, Schepis T, Koepfli P, et al. Accuracy of 64-slice CT angiography for the detection of functionally relevant coronary stenoses as assessed with myocardial perfusion SPECT. Eur J Nucl Med Mol Imaging. January 12, 2007 [Epub ahead of print].

19. Schepis T, Gaemperli O, Koepfli P, et al. Comparison of 64-slice CT with gated SPECT for evaluation of left ventricular function. J Nucl Med. 2006;47: 1288-1294.

20. Schepis T, Gaemperli O, Koepfli P, et al. Use of coronary calcium score scans from stand-alone multislice computed tomography for attenuation correction of myocardial perfusion SPECT. Eur J Nucl Med Mol Imaging. 2007;34:11-19.
21. Koepfli P, Hany TF, Wyss CA, et al. CT attenuation correction for myocardial perfusion quantification using a PET/CT hybrid scanner. J Nucl Med. 2004;45: $537-542$.

22. Fricke H, Fricke E, Weise R, Kammeier A, Lindner O, Burchert W. A method to remove artifacts in attenuation-corrected myocardial perfusion SPECT introduced by misalignment between emission scan and CT-derived attenuation maps. J Nucl Med. 2004;45:1619-1625.

23. Germano G, Kavanagh PB, Waechter P, et al. A new algorithm for the quantitation of myocardial perfusion SPECT. I. Technical principles and reproducibility. J Nucl Med. 2000;41:712-719.

24. Berman DS, Kiat H, Friedman JD, et al. Separate acquisition rest thallium-201/ stress technetium-99m sestamibi dual-isotope myocardial perfusion singlephoton emission computed tomography: a clinical validation study. J Am Coll Cardiol. 1993;22:1455-1464.

25. Fleischmann S, Koepfli P, Namdar M, Wyss CA, Jenni R, Kaufmann PA. Gated ${ }^{99 m}$ Tc-tetrofosmin SPECT for discriminating infarct from artifact in fixed myocardial perfusion defects. J Nucl Med. 2004;45:754-759.

26. Cerqueira MD, Weissman NJ, Dilsizian V, et al. Standardized myocardial segmentation and nomenclature for tomographic imaging of the heart: a statement for healthcare professionals from the Cardiac Imaging Committee of the Council on Clinical Cardiology of the American Heart Association. Circulation. 2002;105: 539-542.

27. Austen WG, Edwards JE, Frye RL, et al. A reporting system on patients evaluated for coronary artery disease: report of the Ad Hoc Committee for Grading of Coronary Artery Disease, Council on Cardiovascular Surgery, American Heart Association. Circulation. 1975;51:5-40.

28. Gibbons RJ, Abrams J, Chatterjee K, et al. ACC/AHA 2002 guideline update for the management of patients with chronic stable angina: summary article-a report of the American College of Cardiology/American Heart Association Task Force on Practice Guidelines (Committee on the Management of Patients with Chronic Stable Angina). J Am Coll Cardiol. 2003;41:159-168.

29. Hachamovitch R, Berman DS, Shaw LJ, et al. Incremental prognostic value of myocardial perfusion single photon emission computed tomography for the prediction of cardiac death: differential stratification for risk of cardiac death and myocardial infarction. Circulation. 1998;97:535-543.

30. Bax JJ, Beanlands RS, Klocke FJ, et al. Diagnostic and clinical perspectives of fusion imaging in cardiology: Is the total greater than the sum of its parts? Heart. 2007;93:16-22.

31. Scheffel H, Alkadhi H, Plass A, et al. Accuracy of dual-source CT coronary angiography: first experience in a high pre-test probability population without heart rate control. Eur Radiol. 2006;16:2739-2747. 